\title{
Interfaces
}

\section{Un herbier d'images : la collection de reproductions photographiques rassemblées par Bourdelle}

\section{Chloë Théault}

\section{(2) OpenEdition}

Journals

Édition électronique

URL : http://journals.openedition.org/interfaces/645

DOI : 10.4000/interfaces.645

ISSN : 2647-6754

Éditeur :

Université de Bourgogne, Université de Paris, College of the Holy Cross

\section{Édition imprimée}

Date de publication : 21 juin 2019

Pagination : 25-43

ISSN : $1164-6225$

\section{Référence électronique}

Chloë Théault, « Un herbier d'images : la collection de reproductions photographiques rassemblées par Bourdelle », Interfaces [En ligne], 41 | 2019, mis en ligne le 21 juin 2019, consulté le 07 janvier 2021. URL : http://journals.openedition.org/interfaces/645; DOI : https://doi.org/10.4000/interfaces.645

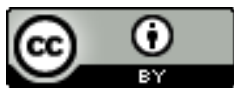

Les contenus de la revue Interfaces sont mis à disposition selon les termes de la Licence Creative Commons Attribution 4.0 International. 


\title{
UN HERBIER D'IMAGES : LA COLLECTION DE REPRODUCTIONS PHOTOGRAPHIQUES RASSEMBLÉES PAR BOURDELLE
}

\author{
Chloë Théault \\ Conservatrice du patrimoine, Responsable des fonds \\ de photographies et de sculptures, musée Bourdelle, Paris
}

«Ce soir j'étudierai Le Jugement dernier de Michel Ange. J’en ai une fort belle photographie. Un sublime épouvantement doit se dégager de l'original de cette reproduction de fresque. » (Bourdelle,

fin des années $1880^{1}$ )

Le musée Bourdelle, situé à Paris, est consacré à l'œuvre du sculpteur Émile Antoine Bourdelle (1861-1929). Créé sur les lieux de vie et de création de l'artiste, ouvert en 1949, le musée conserve 2500 bronzes, 2400 plâtres, des terres, quelques marbres, 140 peintures, 7200 dessins, des moules, des objets personnels de l'artiste, 15000 photographies - majoritairement de ses œuvres - et de très nombreux documents d'archives. Parmi ceux-ci, on compte environ 2500 cartes postales et 1300 reproductions photographiques d'œuvres d'art que Bourdelle a rassemblées au fil de sa vie. Ce corpus témoigne non seulement de la diffusion de la photographie au tournant du XIX ème siècle, mais il nous pousse également à nous interroger sur la place occupée par la reproduction photographique dans la formation et dans le processus créatif du sculpteur. Quel intérêt un artiste qui travaille le volume peut-il en effet trouver dans une reproduction plane, en deux dimensions, impliquant un point de vue unique, imposé ?

Lettre de Bourdelle au banquier Hippolyte Lacaze, sans date, mais dans laquelle il évoque la mort de sa mère survenue en janvier 1888 et que l'on peut donc supposer avoir été écrite à la fin des années 1880 (cote AB/B.2.05.C36.02, archives du musée Bourdelle). 
Le sujet de l'impact des reproductions photographiques sur l'acte créatif n'est pas neuf : plusieurs études ont déjà été consacrées aux liens entre photographie et peinture, ainsi chez Bonnard ou Delacroix, ou entre photographie et sculpture, ainsi chez Rodin, Brancusi, Maillol ou encore Giacometti $^{2}$. Mais le corpus des reproductions photographiques et des cartes postales de Bourdelle, qui frappe par son ampleur et sa cohérence - même si l'on peut craindre des pertes au fil du temps, avant que le projet de musée et de rassemblement des archives n'ait été finalisé - n'a jusqu'alors jamais été étudié dans sa globalité et nous offre un angle de recherche nouveau. En effet, si selon Véronique Gautherin « il ne semble pas que [l'usage de la photographie] ait jamais été source directe d'étude, en tout cas revendiquée » (Gautherin 19), la présente étude interroge pour sa part non pas la pratique photographique mais ses résultantes, à savoir les multiples reproductions photographiques qui ont inondé les ateliers d'artistes dès le XIX ${ }^{\text {ème }}$ siècle. Il s'agit alors d'interroger la façon dont ces reproductions ont pénétré le quotidien comme l'univers créatif de Bourdelle. Ces images ont-elles été de simples vignettes rassemblées pour le plaisir de l'œil, ou ont-elles joué un rôle dans sa formation comme dans sa pratique artistique? Et pourquoi Bourdelle semble-t-il avoir décidé d'un ordonnancement de ces reproductions, puisque d'un côté l'on trouve des cartes postales précautionneusement rassemblées au sein d'albums et, de l'autre, des reproductions souvent laissées éparses ? S'agit-il là d'une simple rationalisation du rangement ou, plus fondamentalement, d'une répartition signifiant des usages différenciés de ces diverses catégories d'images? Autant de questions auxquelles l'analyse qui suit va tenter de répondre.

\section{La bosse et la ronde-bosse comme sources de formation et d'inspiration}

La formation des artistes au XIX ${ }^{\mathrm{eme}}$ siècle reposait en partie sur l'utilisation de la reproduction en volume. Bourdelle a ainsi travaillé le dessin d'après les moulages en bosse et en ronde-bosse de l'École municipale de dessin de Montauban, sa ville natale, puis lors de son passage à l'École des Beaux-Arts de Paris (1884-1886), où avait été créée une importante gypsothèque permettant d'exposer, dans la cour et le Palais des études, des éditions en plâtre destinées à l'enseignement du dessin et l'étude de la sculpture ${ }^{3}$. Bourdelle a aussi pu avoir connaissance de moulages d'œuvres lors de ses visites de musées, comme celui de Montauban qui comptait une salle des plâtres et des antiques, le Musée

Nous pourrons nous référer aux ouvrages suivants : Baldassari ; Brown ; Frizot, Frohne, Malsch et al. ; Hodgson ; Johnson (1995 ; 1998) ; Kosinski ; Marcoci ; Mason, Pinet et Wölfflin ; Richardson, Heiferman, Hayes Tucker, Caws et Combalia ; Teja Bach ; Vaisey (1982); Wood et Curtis.

Nous connaissons la liste des moulages de cette gypsothèque grâce au guide d'Eugène Müntz de 1889, disponible sur Gallica: http://gallica.bnf.fr/ark:/12148/bpt6k1170354x.r=muntz\%20guide?rk=21459;2 
de Sculpture comparée du Palais du Trocadéro qui a ouvert en 1887, ou encore le Louvre - où était d'ailleurs installé un musée des moulages de 1898 à 1927 - dont Bourdelle était un visiteur régulier, puisqu'il s'y rendait « tous les matins » ainsi qu'il l'a écrit en 19194. En octobre 1903, Bourdelle a même rendu compte à son ami et grand critique d'art Elie Faure de sa visite d'un musée de moulages situé à Bruxelles; dans ce «musée de débris », ainsi qu'il l'a qualifié, il a notamment découvert un fragment du temple de Zeus à Olympie qui l'a fortement impressionné ${ }^{5}$.

On comprendra alors que Bourdelle n'ait pas manqué au fil de sa vie de rappeler l'importance des moulages pour la formation de son œil. Suite à sa visite de l'Exposition coloniale à Marseille de 1922, il a ainsi écrit à sa fille Rhodia à propos des " sublimes moulages grecs » : " On ne peut rien voir de plus admirable. Ce sont là les sculptures grecques les plus belles qu'on puisse voir. Il ne peut y avoir rien de plus beau en Grèce même $»^{6}$. À sa femme Cléopâtre, il a aussi écrit, à la même époque : «Il y a là aussi d'ardents moulages grecs. [...] Ces plâtres [...] sont les plus belles œuvres des Grecs $»^{7}$. Notons ici que Bourdelle n'établissait aucune distinction entre le moulage et l'original, entre la copie et l'œuvre première : les uns comme les autres étaient pour lui des répertoires de formes où puiser son inspiration.

Ce goût explique qu'à l'instar d'Auguste Rodin - qu'il a aidé à enrichir sa collection d'antiques à partir du début des années 1890 - Bourdelle a rassemblé sa propre collection d'antiques et de moulages antiques ou médiévaux. Celle-ci rassemblait par exemple une tête de Kouros du Ptoïon, des tanagras - certaines étant toutefois des créations modernes mises sur le marché pour répondre au goût d'alors - et des moulages d'œuvres d'époque médiévale, comme les deux estampages du David luttant contre Goliath de la cathédrale de Reims ${ }^{8}$. Il avait vraisemblablement acquis ces derniers en 1908, pour l'un chez un antiquaire et pour l'autre sur le chantier de restauration de l'édifice, alors qu'il y emmenait ses élèves pour les sensibiliser à la grandeur de la cathédrale dont il était un fervent admirateur.

« J'ai la joie d'être seul au Louvre tous les matins », lettre aux Roussel-Despierres (10 juillet 1919). Archives du musée Bourdelle (Paris), cote AB/B.3.10.063.

5. Musée non identifié. Lettre adressée à Elie Faure le 19 octobre 1903. Archives du musée Bourdelle (Paris), cote AB/B.2.04.C5.21.

6. Lettre adressée à sa fille Rhodia le 18 octobre 1922. Archives du musée Bourdelle (Paris), cote AB/B.2.10.1.C1.30. Lettre adressée à sa femme Cléopâtre, sans date. Archives du musée Bourdelle (Paris), cote AB/B.3.11. Ils sont conservés au sein des collections du musée Bourdelle, numéros d'inventaire MBCO.17 et MBCO.18. 


\section{Reproductions photographiques et cartes postales : le monde et l'art en deux dimensions}

De la cathédrale de Reims, Bourdelle avait aussi rassemblé plusieurs centaines de reproductions photographiques, majoritairement des cartes postales. Si le volume total de sa collection de reproductions photographiques, estimé à 3800 images, est conséquent, il est toutefois impossible et vain d'en dresser la liste complète. Des reproductions ont certainement été perdues, et a contrario certaines ont pu avoir été ajoutées par sa veuve et sa fille après sa mort, lorsqu'elles se sont attelées à promouvoir l'œuvre mémorielle du maître, ce qui vit son aboutissement dans l'ouverture du musée.

Quelques sources et indices permettent toutefois de savoir ce que Bourdelle avait vu ou collectionné lui-même. Citons en premier lieu les archives écrites et les photographies sur lesquelles il est parfois possible de repérer la présence de reproductions punaisées aux murs. Il est utile aussi de prêter attention aux preuves d'usage, telles que les traces de terre, les annotations, les marouflages sur supports en carton, ou encore les systèmes d'attache. Des déductions sont également possibles : à propos de sa vue d'Éleusinion, le temple dédié à Déméter situé sur l'Acropole d'Athènes, Bourdelle a précisé qu'elle a été « réalisée le 22 juin 1921 à Versailles », ce qui indique qu'elle a été faite d'après une reproduction photographique, et non in situ' ${ }^{9}$. Enfin, les copies d'œuvres réalisées par Bourdelle sont d'autres sources d'information sur la place occupée par les reproductions photographiques. Certaines de ses copies peuvent en effet avoir été faites devant des œuvres originales, comme le dessin du Niobide se retirant une flèche $d u$ thorax ${ }^{10}$, d'après une œuvre conservée au musée du Louvre ; et enfin des dessins et une peinture semblent aussi avoir été réalisés d'après des œuvres de la collection personnelle de Bourdelle, qu'il s'agisse indifféremment d'antiques ou de copies ${ }^{11}$. À contrario, d'autres copies ont vraisemblablement trouvé leur inspiration dans des reproductions photographiques, comme cela a pu être le cas pour ses copies d'œuvres orientales ${ }^{12}$. De même, si l'une des copies que Bourdelle a réalisée du Mö̈se de Michel-Ange peut se rapprocher des images diffusées par Alinari ou par Brogi, et dont Bourdelle possédait deux cartes postales, ce n'est pas le cas de la seconde copie, dont le point de vue ne

Collection du musée Bourdelle, numéro d'inventaire MBD.3173.

10. Bourdelle, Panticapée, copie d'après Niobide se retirant une flèche du thorax (collection du musée Bourdelle, numéro d'inventaire MBD.3257).

11. La Peinture d'un tanagra (collection du musée Bourdelle, numéro d'inventaire MBP.018) semble ainsi représenter l'une des tanagras de la collection de Bourdelle, et une photographie sur plaque de verre garde la mémoire d'un dessin de Bourdelle représentant une koré qui pourrait être celle de sa collection (collection du musée Bourdelle, numéro d'inventaire MBPV.4452).

12. Bourdelle, Copies d'œuvres orientales (collection du musée Bourdelle, numéros d'inventaire MBD.3229, MBD.4536). 
colle à aucune des quatre reproductions présentes dans la collection de Bourdelle (Figure 1); on peut en conclure soit qu'il a utilisé, pour faire cette copie, une autre reproduction qui a depuis été perdue, soit qu'il s'est posté devant le moulage de l'œuvre conservé à l'École des Beaux-Arts. Cet exemple montre toute la difficulté de ce jeu de piste visant à retrouver ce que Bourdelle avait vu et, par là, ce qui influençait son regard.

\section{La collection de cartes postales : un monde en miniature}

Parmi l'ensemble des reproductions photographiques, le fonds du musée Bourdelle compte 2100 cartes postales rassemblées en vingt-et-un albums, pour la plupart thématiques. On peut ajouter à ce chiffre environ quatre cents cartes volantes - ayant peut-être été retirées des albums au fil des années sans y avoir ensuite été réintégrées. Certaines de ces cartes ont été adressées à la tante de Bourdelle, à sa première épouse Stéphanie, à sa seconde épouse Cléopâtre ${ }^{13}$, ou à d'autres personnes de son entourage, notamment ses praticiens. Mais à lire ces cartes, il apparaît qu'elles étaient souvent adressées à plusieurs personnes et donc qu'elles circulaient entre plusieurs mains. Il est donc fort possible que Bourdelle ait vu leur très grande majorité, ce qui justifie qu'elles aient toutes été incluses dans cette analyse.

13. Une grande partie des cartes postales envoyées de l'étranger lui étaient adressées.

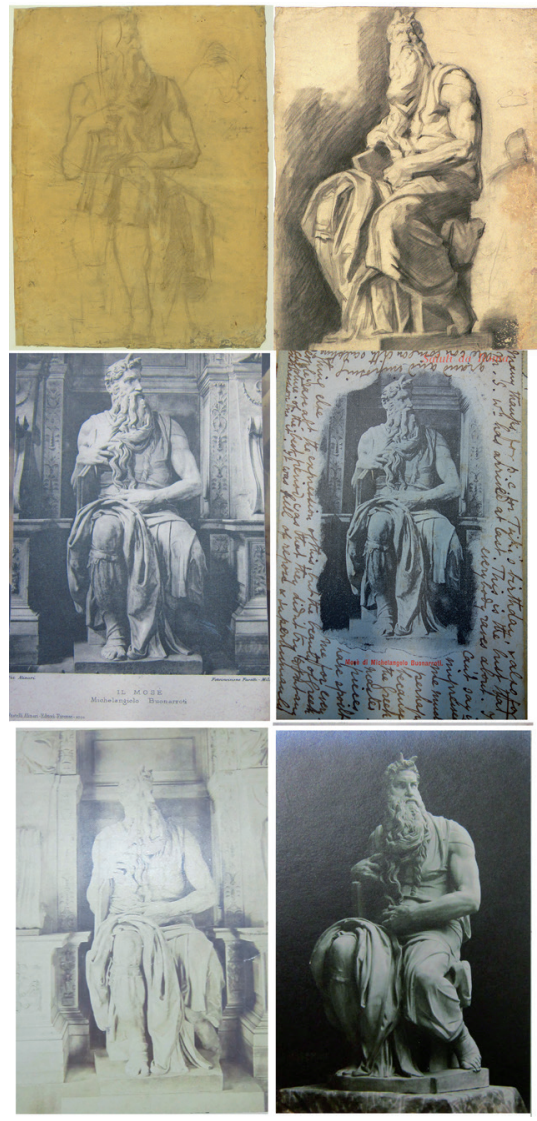

Figure 1 : Comparaison de quatre photographies du Moïse de Michel-Ange présentes dans la collection de Bourdelle et de deux copies réalisées par Bourdelle, l'une l'ayant été d'après une carte postale de la maison Alinari ou d'après une photographie du studio Brogi (en haut à gauche, collection du musée Bourdelle, Paris, numéro d'inventaire MBD.1405) et l'autre d'après une source non identifiée (en haut à droite, collection du musée Bourdelle, Paris, numéro d'inventaire MBD.6251) 
Cet important volume de cartes postales n'a rien de surprenant. Au début du vingtième siècle, la diffusion et la vente de cartes postales ont connu un grand essor. Elles étaient un support de communication très répandu et très fréquent - la poste pneumatique permettait de transmettre un message en quelques dizaines de minutes à Paris. Liées au développement du tourisme et de la publicité, elles renvoyaient des images des différentes régions et activités de France, et toutes sortes de sujets se voyaient traités. Cet universalisme de la carte postale se retrouve dans la collection de Bourdelle, qui regroupe une majorité de reproductions de monuments ${ }^{14}$, de statuaire ${ }^{15}$ et d'autres œuvres d'art ${ }^{16}$, mais aussi des panoramas urbains ${ }^{17}$, des vues associant urbanité et nature ${ }^{18}$, des paysages naturels ${ }^{19}$, des vues pittoresques d'ailleurs regroupées dans un album spécifique ${ }^{20}$, quelques images de la vie moderne, tels que les ponts, les moyens de transport ou certains événements liés à l'actualité ${ }^{21}$, et enfin une poignée de vues diverses, difficiles à classer.

Pourtant, tout, dans ce corpus, n'est pas représenté à parts égales. Les cartes postales liées à la région natale de Bourdelle - le Tarn - sont en grand nombre ${ }^{22}$, ainsi que celle liées à la Grèce, le pays d'origine de sa seconde épouse et grand sujet d'inspiration et d'admiration esthétique pour lui ${ }^{23}$. Viennent ensuite des cartes postales liées à ses voyages (en Bretagne, Normandie, Angleterre et à Marseille) et à ses lieux de travail (Allemagne, Belgique). A contrario, on remarque la faible proportion d'éléments sur Paris et l'Ile-de-France ${ }^{24}$ ou sur la vie moderne. Ces dernières vues, notons-le aussi, sont disséminées dans les albums au lieu d'être rassemblées, signifiant là qu'il n'y avait pas de volonté d'en constituer un corpus. En ce sens, on peut dire que la collection de cartes postales de Bourdelle visait à rassembler le lointain et le rêvé, plutôt que le proche et le quotidien.

Regardons maintenant les cartes postales représentant des œuvres d'art. Elles témoignent de l'intérêt très fort de Bourdelle pour le construit, le monument, et la statuaire afférente aux monuments. En revanche, peu de cartes postales représentent des sculptures autonomes, entendons par là qui ne

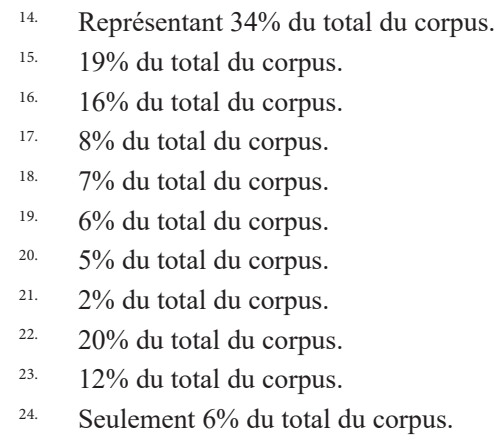


sont pas des éléments d'architecture. Celles-ci semblent d'ailleurs le plus souvent avoir été envoyées à Bourdelle par des amis. Ainsi, Paul-Louis Couchoud lui a écrit : « Puisque le léger sourire de cette petite déesse vous a plu, laissez-moi vous l'envoyer comme une messagère ${ }^{25}$; et François Monod a pour sa part écrit le 8 novembre 1928 : «Le Musée du Cinquantenaire a deux ou trois beaux antiques qui méritent l'attention. Voici le plus précieux, une admirable tête dont le caractère et la date sont indiqués ci-dessous. Quelles beautés de modelé, et quel métier de marbrier ! ${ }^{26}$ (figure 2).
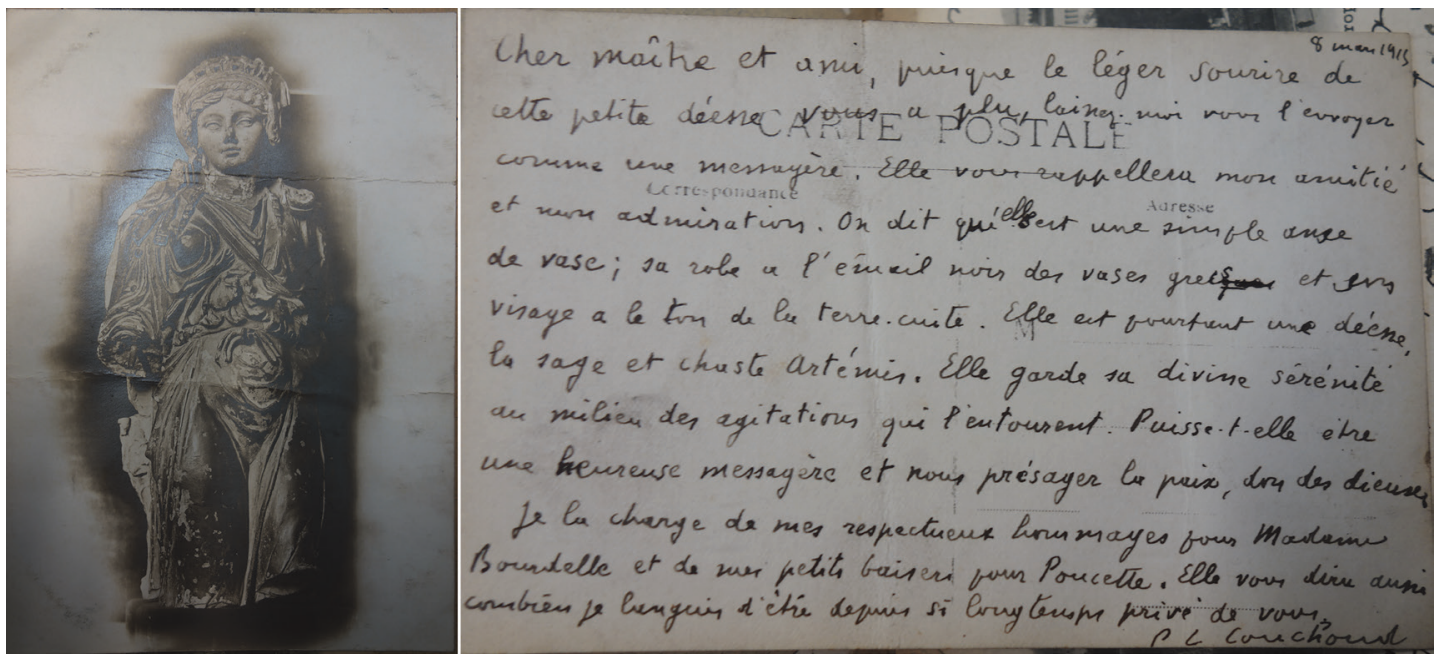

Figure 2 : Recto et verso d'une carte postale envoyée par Paul-Louis Couchoud à Bourdelle le 8 mars 1915 (collection du musée Bourdelle)

Certains des albums de cartes postales ont été titrés de la main de Bourdelle, nous révélant ainsi l'ordonnancement qu'il avait délibérément choisi, et par là l'importance qu'il leur accordait. Des albums étaient ainsi consacrés aux « Archaïques grecs », aux chapiteaux, aux stalles de Rouen,

25. Paul-Louis Couchoud, médecin, philosophe et poète, était l'époux de la belle-sœur de Bourdelle. Carte postale datée du 8 mars 1915, collection du musée Bourdelle.

26. François Monod était conservateur adjoint au musée du Luxembourg. Carte postale datée du 8 novembre 1928, collection du musée Bourdelle. 
ou encore à l'art gothique. D'autres albums, bien que non annotés sur leur tranche, ont été constitués par thématiques : les églises pour l'un, Chartres ou la cathédrale de Reims pour d'autres. Soulignons aussi que plusieurs de ces albums rassemblent des lots de cartes postales encore vierges, et qui n'ont donc jamais été utilisées pour leur fonction première de supports d'écriture. C'est le cas pour un lot de quarante-quatre cartes portant sur la statuaire de la cathédrale de Chartres ${ }^{27}$, pour un autre lot de quatrevingt-quatre cartes sur les stalles de la cathédrale de Rouen, ou enfin pour un lot de quatre-vingt-dix-huit cartes sur les médaillons de cette même cathédrale. Notons aussi que, très symptomatiquement, les albums rassemblent majoritairement des reproductions vierges : par exemple, l'album rassemblant cent cartes sur l'art gothique n'en compte que vingt-deux écrites. On peut en conclure que l'image primait sur la valeur messagère de la carte. C'est sans doute aussi pourquoi l'ordonnancement des cartes a été effectué d'après leur iconographie, et non, ce qui aurait pu être une autre approche, selon les expéditeurs.

Une analyse quantitative de ce corpus révèle par ailleurs la prépondérance de l'art du Moyen Âge (principalement roman), qui en occupe en effet presque la moitié ${ }^{28}$ - et c'est aussi lui qui a fait l'objet de la plus grande attention classificatoire de Bourdelle. Viennent ensuite l'art de la Renaissance ${ }^{29}$ et l'art antique ${ }^{30}$. Les reproductions d'œuvres picturales sont pour leur part en très faibles proportions ${ }^{31}$, et les albums comportent peu de contemporains de Bourdelle, sinon Auguste Rodin et Paul Gauguin ${ }^{32}$.

Enfin, la lecture de ces cartes révèle aussi l'importance qu'elles recelaient aux yeux de Bourdelle. Au dos de l'une d'elles, une de ses correspondantes lui a ainsi écrit : « Cher Maître, voulez-vous ajouter à votre collection cette humble porte romane... » ${ }^{33}$. Preuve, donc, que ces albums constituaient véritablement une collection qui lui permettait de garder la mémoire de certaines œuvres. Cléopâtre a aussi écrit à Rose, la tante de Bourdelle : « Bourdelle demande que vous lui gardiez cette carte qui est l'ingrate reproduction d'une admirable mosaïque de Torcello », ou encore : « Ne perdez pas cette carte, Bourdelle y tient ${ }^{34}$ (Figure 3). Enfin, au dos d'une carte se déchiffre l'écriture de Bourdelle

\footnotetext{
Éditeur Houvet.

Il représente en effet $48 \%$ du total du corpus.

$19 \%$ du total du corpus.

$11 \%$ du total du corpus.

$3 \%$ de peintures du XVII ${ }^{\text {ème }}$ siècle, $4 \%$ de peintures du XVIII ${ }^{\text {ème }}$ siècle.

32. Le corpus d'œuvres du XIX ${ }^{\text {ème }}$ siècle représente $12 \%$ du total, mais ce chiffre est gonflé par la présence massive de reproductions d'œuvres de Bourdelle.

33. Carte postale écrite par Madelaine Mouriès (collection du musée Bourdelle).

34. Carte datée de 1922 (collection du musée Bourdelle).
} 
et l'annotation «Collection E... » (mile?) ${ }^{35}$, ce qui laisse à nouveau penser qu'il avait constitué une collection de cartes postales dans laquelle il allait parfois piocher.
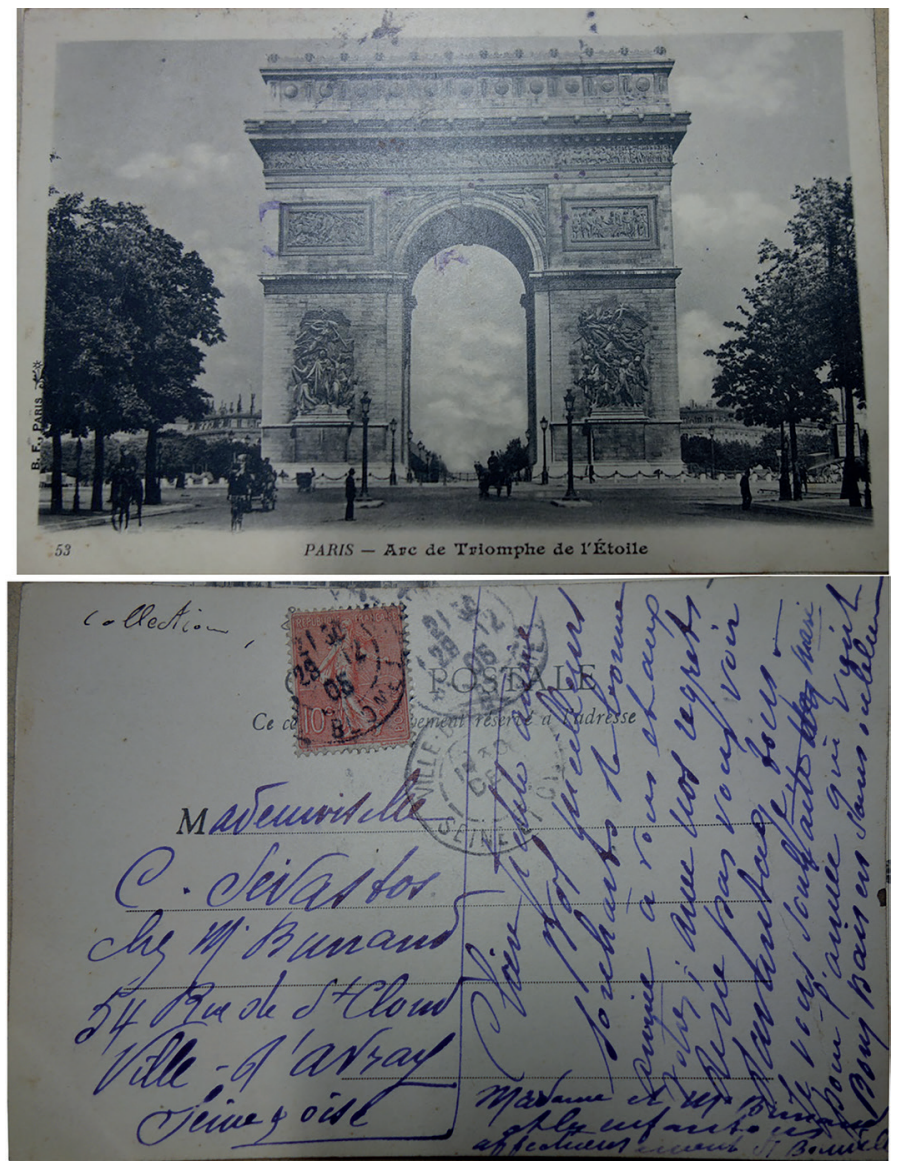

Figure 3 : Recto et verso d'une carte postale envoyée par Bourdelle à Cléopâtre en 1905 (collection du musée Bourdelle) 
Et pourtant, chose étonnante, il n'a pas été trouvé d'exemple de copies que Bourdelle aurait réalisées directement d'après des cartes postales. De manière générale ces cartes postales semblent donc avoir été des objets de collection permettant à Bourdelle de se constituer un panorama imagé et miniature du monde qui lui tenait à cœur, ou bien des objets de relation témoignant de son réseau amical et familial, mais elles ne semblent pas avoir été des outils de travail pour parfaire sa main.

\section{Les reproductions photographiques : un musée de poche}

Les cartes postales n'étaient pas les seules reproductions photographiques détenues par Bourdelle. En effet, le fonds du musée comporte également environ 1300 reproductions pour la plupart éparses. Des séries peuvent néanmoins être repérées, comme celle sur Pompéi ou une autre sur l'art grec cette dernière étant le résultat du travail photographique d'Aristote Rhomaïdès et Papayannopoulos vers 1900. Certaines reproductions portent parfois la signature de photographes tels que Michelez ou de maisons d'éditions photographiques comme Alinari ou Calavas. Notons aussi qu'une grande part des reproductions sur la Grèce, ainsi la série de photographies signées de Frédéric Boissonnas et sans doute faites entre 1907 et 1919, ont vraisemblablement appartenu à Cléopâtre. Il convient donc de toujours garder à l'esprit que toutes les reproductions n'ont pas forcément été collectionnées par Bourdelle lui-même.

L'analyse quantitative de ce corpus permet de noter qu'outre les vues de paysages, qui représentent une petite part du total du corpus ${ }^{36}$, la majorité des reproductions sont celles d'œuvres d'art, à la grande différence des cartes postales : il n'y a donc pas la même volonté universaliste que dans sa collection de cartes postales. Un autre point d'étonnement concerne la très forte représentation de l'art antique, et notamment grec, qui occupe environ la moitié du corpus ${ }^{37}$. Viennent ensuite le Moyen Âge ${ }^{38}$ et la Renaissance, avec une forte représentation de Michel-Ange ${ }^{39}$. Les contemporains de Bourdelle, tels que Jules Dalou, Théodore Chassériau ou Jean Carriès, sont sensiblement plus représentés que dans les cartes postales ; ils ont pour la plupart été rassemblés dans un corpus de reproductions appelé « Imagier » comportant une trentaine de reproductions photographiques parues dans la presse. Enfin,

\footnotetext{
$7 \%$ du total du corpus.

$47 \%$ de l'ensemble des reproductions.

$16 \%$ du total du corpus.

$10 \%$ du total du corpus.
} 
on note aussi la présence de reproductions d'œuvres extra-occidentales ${ }^{40}$, ce qui n'apparaissait que peu dans les cartes postales. Il apparaît donc que ces deux sous-ensembles que sont les cartes postales d'une part et les reproductions éparses d'autre part, avaient des visées bien distinctes : les cartes postales visaient à témoigner d'une vision du monde dans son acception la plus large, tandis que les reproductions photographiques constituaient le «musée de poche » de Bourdelle dans lequel il allait puiser.

Il est alors intéressant de questionner l'évolution de ce répertoire d'images. Si nous n'avons que peu d'informations sur la façon dont Bourdelle a acquis ces reproductions, on sait toutefois que dans les années 1880 - la date ayant pu être déduite de dessins exécutés au dos des cartons de montage sur lesquels ces photographies avaient été marouflées - Bourdelle possédait des reproductions d'œuvres principalement de la Renaissance italienne, comme les reproductions du David, du Moïse ou encore du Jugement dernier de Michel-Ange. De telles reproductions étaient alors largement diffusées par les maisons d'édition et par les librairies spécialisées - que Bourdelle fréquentait, par exemple la maison Pourchet - jusque dans les ateliers de l'École des Beaux-arts, puisque le coût peu élevé et le maniement aisé de ces reproductions en faisaient des outils de travail accessibles et pratiques pour les élèves.

Au fil des années, le musée de poche de Bourdelle a ensuite intégré des reproductions d'œuvres antiques et extra-européennes, cela au gré de ses visites et voyages. Ainsi sa collection d'images s'est-elle enrichie de l'art égyptien, mais aussi de l'art indonésien qu'il a vu à l'Exposition de 1900, de l'art cambodgien dont il a eu connaissance à Marseille lors de l'exposition coloniale de 1906, de l'art indien vu à Londres en 1912, à l'Indian Museum, et de l'art khmer, qu'il a pu voir à Marseille en 1922. Notons que les reproductions de sculptures indonésiennes circulaient en grand nombre à l'époque, aussi n'y a-t-il rien de surprenant à les trouver dans sa collection. Surtout, cette diffusion de l'art extra-européen par la photographie signale une évolution des regards et de l'exercice même de la pensée chez les artistes qui ont ainsi vu leurs horizons de références s'élargir considérablement - un point mis en exergue par André Malraux dans le Musée imaginaire (1947).

L'analyse comparative de ces deux corpus met aussi en lumière une autre différence d'importance, à savoir que Bourdelle n'a pas réservé les mêmes usages aux cartes postales et aux reproductions photographiques. Plusieurs traces d'usage indiquent que les reproductions étaient très présentes dans son quotidien : trous d'épingles, traces de terre sur les revers, retouches, présence de systèmes d'attache ou de pâte à fixer. Par ailleurs, l'étude des détails des photographies représentant les espaces de vie et de travail de Bourdelle permet là aussi de repérer la présence de reproductions photographiques sur les murs, ce qui ne semblait pas être le cas des cartes postales (Figure 4). Les archives témoignent 
elles aussi de cette différence d'utilisation entre cartes postales et reproductions. Ainsi, Bourdelle écrit à son ami le banquier Hippolyte Lacaze : «Ce soir j'étudierai Le Jugement dernier de Michel Ange. J'en ai une fort belle photographie ${ }^{41}$. Bourdelle collectionnait les cartes postales, mais il étudiait les reproductions. Cela explique alors sans doute pourquoi il a été possible de retrouver les sources de certaines des copies faites par Bourdelle, qui s'avèrent être toutes des reproductions photographiques et jamais des cartes postales : ainsi, sa copie de l'Ugolin de Carpeaux a été réalisée d'après une photographie de Charles Marville du groupe sculpté (Figure 5).

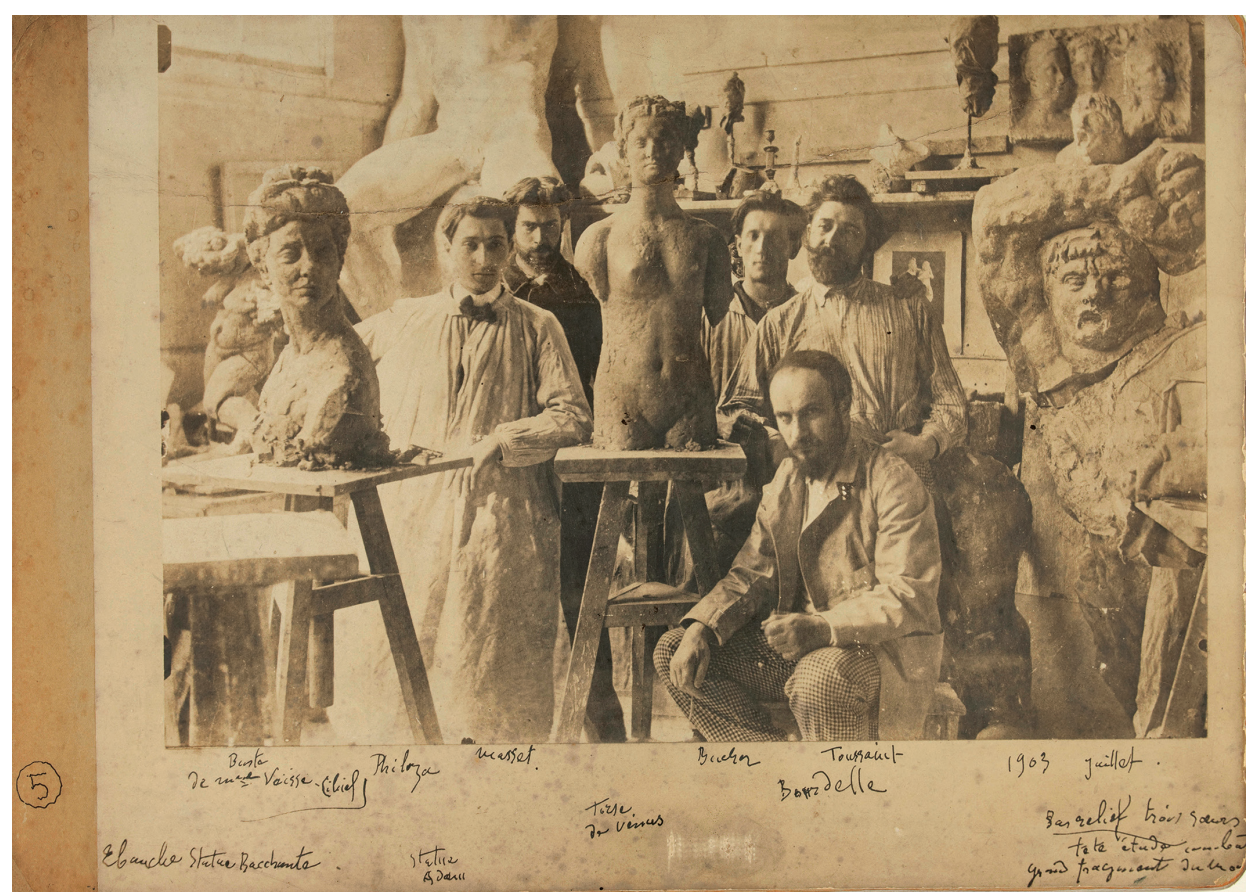

Figure 4 : Photographie anonyme montrant Bourdelle et ses praticiens dans son atelier, 1903 (collection du musée Bourdelle, numéro d'inventaire MBPH.273). Punaisée sur le mur se trouve la reproduction par le studio Brogi du Groupe de Pan et Apollon du musée de Naples, présente dans la collection de reproductions de Bourdelle. 

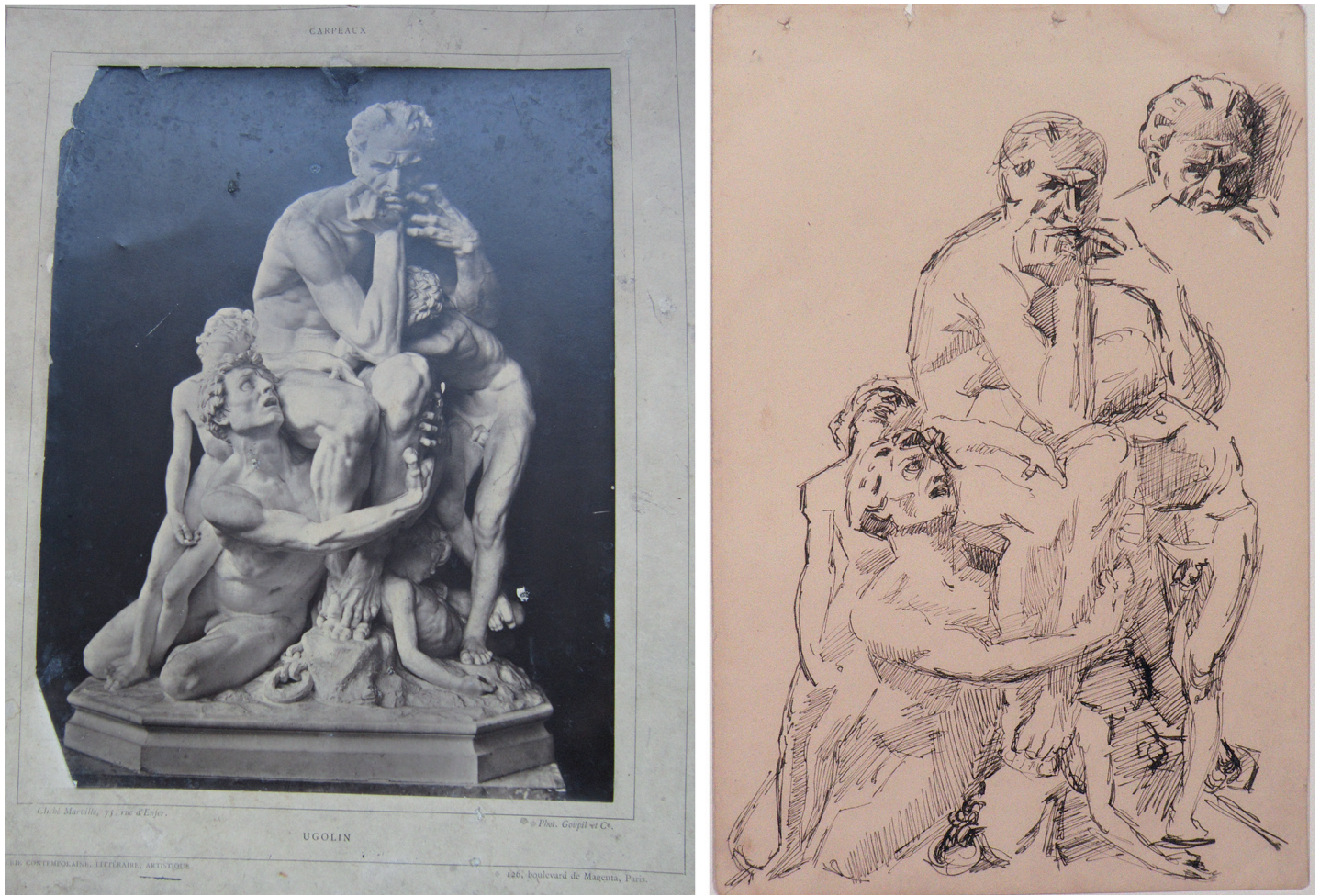

Figure 5 : Comparaison de la photographie de Charles Marville de l'Ugolin de Carpeaux, provenant de la collection de Bourdelle, et de la copie réalisée par Bourdelle (collection du musée Bourdelle, numéro d'inventaire MBD.6252)

\section{La bibliothèque : le royaume des pairs}

Dans cette étude, ce sont peut-être les absences qui surprennent le plus. En effet, Bourdelle a souvent souligné son intérêt pour certains de ses contemporains, comme Jean-Auguste-Dominique Ingres, Pierre Puvis de Chavannes, ou encore Paul Cézanne. S'ils ne sont pas totalement absents, ils sont 
pourtant très peu représentés au sein du corpus de reproductions photographiques et de cartes postales. Sa connaissance de l'art de son temps semble lui provenir d'autres sources, non seulement ses visites de musées et de Salons et ses rencontres, mais aussi son abondante bibliothèque.

Si l'inventaire est encore en cours afin de définir avec certitude quels étaient les livres détenus par Bourdelle - là encore des faisceaux d'indices peuvent être convoqués, ainsi les dates de parution, les annotations de sa main, la présence de son monogramme sur les pages de garde, les mentions dans la correspondance, les dédicaces - on peut toutefois avancer quelques informations. Sa bibliothèque, qui rassemblait environ 670 ouvrages $^{42}$, se divisait en trois grandes catégories : la littérature, l'art, les essais. Presque la moitié des livres sont en effet des ouvrages de littérature ${ }^{43}$, souvent d'ailleurs écrits en langue d'oc, et la poésie y est prépondérante ${ }^{44}$; n'oublions pas que Bourdelle écrivait lui-même beaucoup de poésie, et un de ses écrits figure d'ailleurs dans sa bibliothèque ${ }^{45}$. À parts égales viennent ensuite les essais, qui portent la marque de son réseau amical ${ }^{46}$, et les ouvrages d'art tels que les biographies, les monographies, les livres techniques, ou encore les catalogues de vente. Notons aussi la présence de nombreux ouvrages sur l'art grec, comme celui sur Douris et les peintres de vases grecs ${ }^{47}$ que Bourdelle a largement annoté et dans lequel il a reproduit au calque une œuvre représentant une coupe grecque ${ }^{48}$, ainsi que d'un album de vues photographiques de Pompéi, sur lequel des dessins de fumées ont été ajoutés au crayon de papier en haut du Vésuve ; nous ignorons la provenance de cet album, mais on sait toutefois que Bourdelle s'est rendu en 1922 à Pompéi, et peut-être l'a-t-il acquis à cette occasion. Citons aussi la présence d'ouvrages sur la danse - autour de la figure d'Isadora Duncan - et sur Beethoven, deux des grandes sources d'inspiration de Bourdelle, ainsi que de quelques rares ouvrages sur l'architecture, qui concernent majoritairement les cathédrales, en premier lieu Reims et Chartres. Sont aussi présentes en grand nombre des éditions allemandes ${ }^{49}$. Or, si Bourdelle a certes été en Allemagne en 1909, où il a d'ailleurs acheté un catalogue sur la Sécession, il ne lisait pas l'alle-

42. Le décompte a été fait en isolant les ouvrages provenant du fonds Bourdelle et édités avant 1930.

43. La littérature occupe environ $45 \%$ du total des ouvrages. On peut citer Tolstoï, Flaubert, Maeterlinck, Baudelaire, Mallarmé.

44. Citons par exemple Champsaur, Moreas, Sully Prudhomme, Quercy, Mistral, Perbosc.

45. Vieux usages français (poème de quinze quatrains), édition La Phalange, 1910.

46. Citons Suarès, Michelet, Krishnamurti.

47. Ouvrage écrit par Edmond Pottier (1909).

48. En l'occurrence une coupe attique à figures rouges représentant Zeus enlevant Ganymède (vers 490-480 av J.-C., Paris, musée du Louvre, numéro d'inventaire G123).

49. Par exemple Archaische Plastik der Griechen, Olympische Kunst, Griechische Vasenmalerei, toutes ces éditions datant des années 1920. 
mand : on peut donc en conclure qu'il possédait ces ouvrages non pas tant pour les textes que pour leurs illustrations. De même, dans sa bibliothèque se trouvaient des catalogues de ventes d'antiques et de pierres gravées ; le fait qu'il ait conservé ces livrets qui ne sont d'aucune valeur commerciale dit quelque chose de l'intérêt qu'il leur portait.

L'étude de cette bibliothèque nous permet alors de savoir avec précision quels artistes Bourdelle a regardés et sans doute aussi étudiés : Michel-Ange, Donatello, Rembrandt, Velasquez, Blake, David, Chardin, Rude, Constable, Ingres, Corot, Barye, Carpeaux, Puvis de Chavanne, Manet, Daumier, Rops, Berthe Morisot, Rodin, Carrière, Cézanne, Monet, ou encore des membres de la Rose Croix et des représentants du futurisme ${ }^{50}$. Cette bibliothèque se révèle ainsi être le lieu où Bourdelle avait rassemblé ses pairs. Tandis que les cartes postales et les reproductions sont du ressort de la collectionune collection de dilettante, voire d'amateur - la bibliothèque se veut, elle, lieu symbolique de l'étude savante, érudite, professionnelle.

\section{Les dossiers d'archives : la mémoire de soi}

Bourdelle a été conscient très tôt de l'importance de conserver et transmettre ses archives pour comprendre son œuvre. Aidé de son épouse Cléopâtre, il a minutieusement classé et ordonnancé ses écrits et ses dessins, et il a également constitué vingt importants dossiers d'archives rassemblant un total de quatre-vingt-dix-huit cahiers, treize chemises et un dossier - les cahiers se recoupant parfois ou, pour certains, rassemblant des sujets hétéroclites. Certains de ces dossiers sont consacrés à l'antiquité ${ }^{51}$, à l'art grec ${ }^{52}$, à Ingres ${ }^{53}$, à Carrière et Suarès ${ }^{54}$ mais aussi à plusieurs des œuvres maîtresses de Bourdelle, ainsi l'Héraklès archer ${ }^{55}$, le monument au Général Alvéar ${ }^{56}$, le monument à Mickiewicz ${ }^{57}$ et le Monu-

50. Nous pouvons souligner la présence d'un ouvrage écrit par Marinetti, qui l'a adressé et dédicacé à Bourdelle en « hommage d'une profonde admiration ».

51. Dossiers $n^{\circ} 1$ et 3 .

52. Dossier $n^{\circ} 9$.

53. Dossier $n^{\circ} 19$.

54. Dossier $n^{\circ} 15$.

55. Dossier $n^{\circ} 4$.

56. Dossier $n^{\circ} 12$.

57. Dossier $n^{\circ} 11$. 
ment de la Pointe de Grave ${ }^{58}$. Deux dossiers sont dédiés à son projet de musée ${ }^{59}$ et deux autres dossiers rassemblent des archives personnelles, sur Cléopâtre et sur Rhodia ${ }^{60}$. Notons ici que quelques-unes des reproductions photographiques rassemblées dans ces dossiers ont servi de modèle à des copies de Bourdelle (Figure 6), ce qui prouve à nouveau que les reproductions étaient véritablement pour lui des supports de travail.
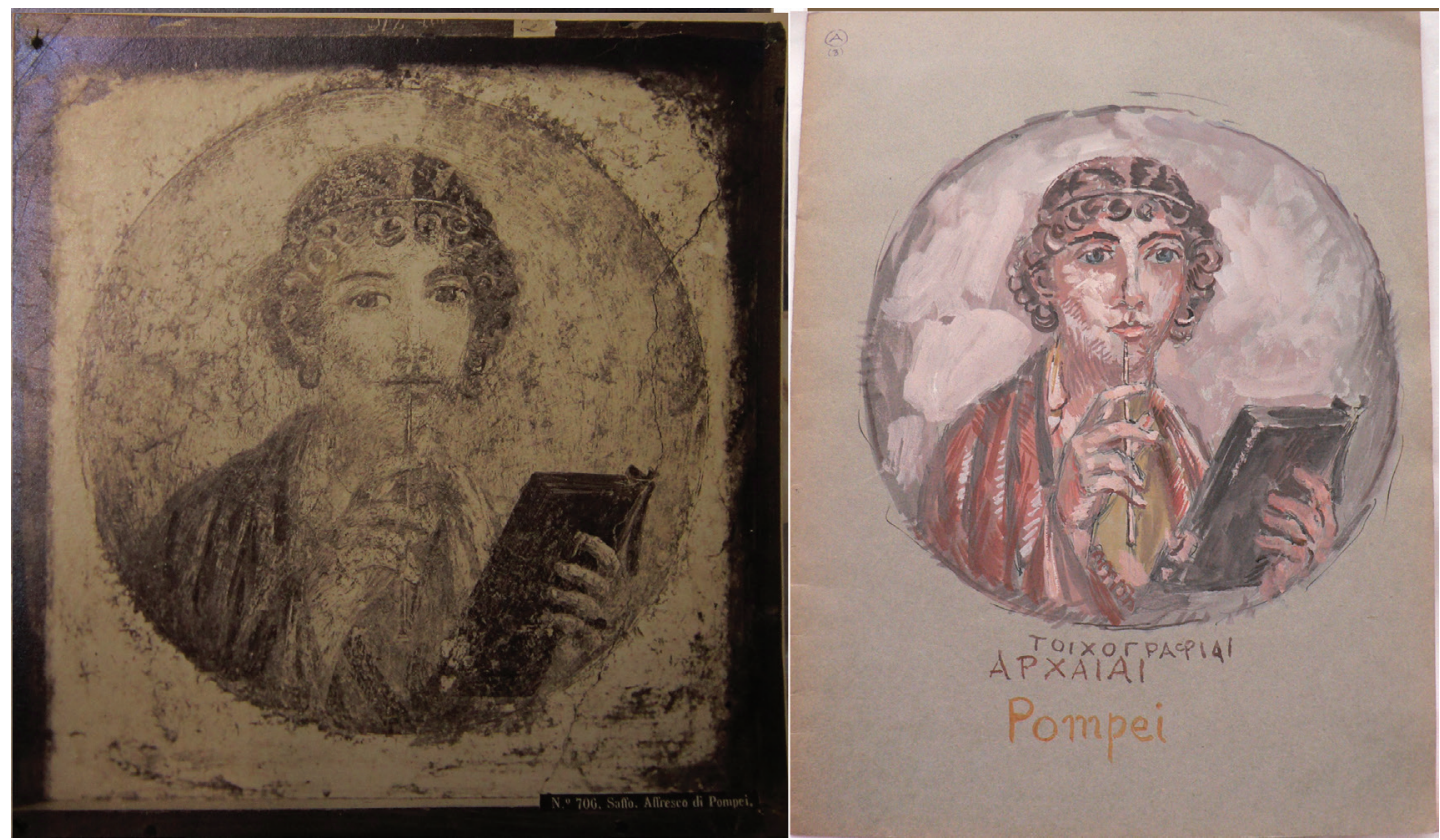

Figure 6 : Comparaison de la reproduction d'une fresque de Pompéi dite Sapho, conservée dans la collection de Bourdelle, et du dessin de couverture du dossier toilé « Pompéi » réalisé par Bourdelle (collection du musée Bourdelle, numéro d'inventaire MBD.4449)

58. Dossier $n^{\circ} 20$.

59. Dossiers $n^{\circ} 17$ et 18 .

60. Dossier $n^{\circ} 16$. 
Mais là encore, il apparaît que ces dossiers d'archives répondent à un registre d'utilisation bien spécifique, distinct de celui des cartes postales, des reproductions éparses et des ouvrages de sa bibliothèque. En effet, presque la moitié des dossiers concernent l'activité de Bourdelle, ses œuvres, ses projets $^{61}$, et une autre grande part portent sur sa vie et ses relations ${ }^{62}$. Ses archives ne témoignent donc pas de son désir de se constituer une miniature du monde ou un musée de poche, même si l'on retrouve la présence de l'art grec et de l'antiquité : elles sont majoritairement centrées sur lui et ses œuvres.

L'art de son temps - qu'il s'agisse d'arts plastiques, de musique ou de littérature - est la deuxième grande catégorie de ces archives ${ }^{63}$, suivi par l'Antiquité ${ }^{64}$. Ces mêmes proportions se retrouvent d'ailleurs lorsque l'on dénombre les seules reproductions ${ }^{65}$. Ces archives oscillent donc entre d'une part le personnel et le contemporain et d'autre part l'antique. Elles sont en ce sens une illustration de l'art de Bourdelle, qui le voulait réitération dans le monde moderne des inspirations antiques.

En conclusion, cet herbier d'images, composé de cartes postales, de reproductions éparses, contenues dans des ouvrages, ou bien encore rassemblées au sein de dossiers d'archives, nous permet de pénétrer l'univers visuel de Bourdelle, de ses goûts comme de ses sources d'inspiration. Il est aussi la parfaite illustration du goût pour l'archive - et ce faisant pour la transmission - si cher à Bourdelle.

\section{OUVRaGes CITÉS}

L'ensemble des documents utilisés provient des collections et des archives du musée Bourdelle.

BALDASSARI, Anne, dir. Brassaï / Picasso. Conversations avec la lumière. Paris : Réunion des musées nationaux, 1999.

BARBILLON, Claire et Stéphanie CANTARUTTI, dir. La Mémoire à l'œuvre. Les archives Antoine Bourdelle. Paris : Paris-Musées, 2009.

BARBILLON, Claire, GODEAU, Jérôme et Amélie SIMIER, dir. Bourdelle et l'antique. Une passion moderne. Paris : Paris-Musées, 2018.

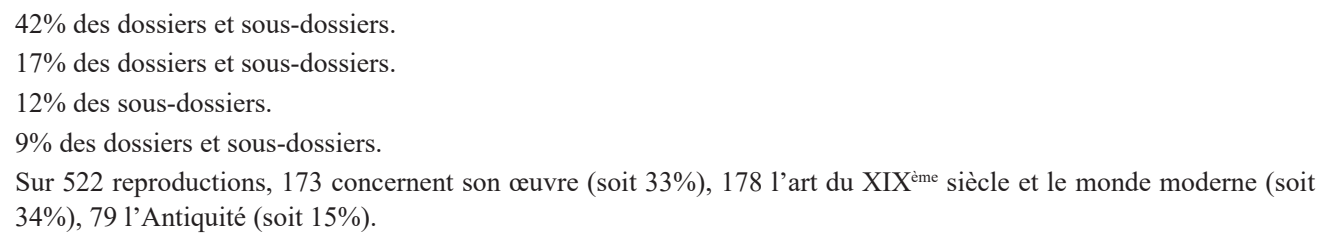


BOISSEROLLES, Claire, FERRAND, Stéphane et Amélie SIMIER, dir. Transmission / Transgression. Maîtres et élèves dans l'atelier : Rodin, Bourdelle, Giacometti, Richier. Paris : Paris-Musées, 2018.

BROWN, Elizabeth. Brancusi Photographs Brancusi. Londres : Thames \& Hudson, 1995.

FRIZOT, Michel, FROHNE, Ursula, MALSCH Friedemann, et al. Lens-Based Sculpture: The Transformation of Sculpture through Photography. Berlin : Akademie der Künste, 2014.

GAUTHERIN, Véronique. L'Eil et la main. Bourdelle et la photographie. Paris : Paris Musées, 2000.

HODGSON, Francis. Rodin, Brancusi, Moore: Through the Sculptor's Lens. Londres : Waddington Custot Galleries, 2015.

JOHNSON, Geraldine. Sculpture and photography: Envisioning the Third Dimension. Cambridge : Cambridge University Press, 1998.

- The Very Impress of the Object: Photographing Sculpture from Fox Talbot to the Present Day. Leeds : Henry Moore Institute, 1995.

KOSINSKY, Dorothy. The Artist and the Camera: Degas to Picasso. New Haven : Yale University Press, 1999.

KRAMER, Hilton. Brancusi: The Sculptor as Photographer. Londres : David Grob Editions / Callaway Editions, 1980.

MALRAUX, André. Musée imaginaire. Genève : Skira, 1947.

MARCOCI, Roxana. The Original Copy: Photography of Sculpture, 1839 to today. New York : Museum of Modern art, 2010.

MASON, Rainer Michael, PINET, Hélène et Heinrich WÖLFFLIN. Pygmalion Photographe : La Sculpture devant la caméra, 1844-1936. Genève : Musée d'art et d'histoire, 1985.

RICHARDSON, John, HEIFERMAN, Marvin, HAYES TUCKER, Paul, CAWS, Mary Ann, et Victoria COMBALIA. Picasso and the Camera. New York : Gagosian Gallery, 2014.

TEJA BACH, Friedrich. Brancusi. Photoreflexion. Paris : Didier Imbert Fine Art, 1991.

THEAULT, Chloë, dir. De bruit et de fureur. Bourdelle sculpteur et photographe. Paris : Le Passage, 2016.

VAIZEY, Marina. The Artist as Photographer. New York : Henry Holt \& co, 1982.

WOOD, Jon et Penelope CURTIS. Close Encounters: The Sculptor's Studio in the Age of the Camera. Leeds : Henry Moore Institute, 2001. 
Résumé : Quel intérêt un artiste qui travaille le volume peut-il trouver dans une reproduction plane, en deux dimensions ? Cette étude vise ainsi à interroger le rôle de la reproduction photographique dans l'univers créatif du sculpteur Bourdelle. Son importante collection, réparties en 2500 cartes postales, 1300 reproductions éparses, à laquelle on doit adjoindre les reproductions contenues dans les 670 ouvrages de sa bibliothèques et les images rassemblées dans ses dossiers d'archives, permet de mettre en lumière quatre registres différenciés d'utilisation. Ainsi, sa collection de cartes postales offre l'image dilettante d'un monde en miniature ; les reproductions éparses constituaient son musée de poche dans lequel il pouvait glaner des outils de travail pour exercer sa main; dans sa bibliothèque, lieu de l'étude et de l'érudition, étaient rassemblés ses pairs; enfin, ses dossiers d'archives visaient à garder la mémoire intime de son œuvre et de sa vie.

Abstract: What interest can a sculptor, who works in volume, find in the use of flat photographic reproductions? This study aims at looking at the role and function of photographic reproductions in Bourdelle's creative process. His personal collection gathers 2500 postcards, 1300 separate reproductions, to which we must add the images included in the 670 books of his library and the reproductions he grouped in his archival folders. This study brings to light four different uses of these images according to their status. Bourdelle's collection of postcards operates as a world in miniature. The separate reproductions constitute a kind of pocket museum that Bourdelle used to make copies. In his library he gathered the books about his peers, who are absent in the first two collections of images. The final category concerns his archival folders which are simply dedicated to the memory of his own works and life. 